\title{
An Ontology-Based Semantic Foundation for Organizational Structure Modeling in the ARIS Method
}

\author{
Paulo Sérgio Santos Jr., João Paulo A. Almeida, Giancarlo Guizzardi \\ Ontology \& Conceptual Modeling Research Group (NEMO) \\ Computer Science Department, Federal University of Espírito Santo (UFES) \\ Vitória, ES, Brazil \\ paulossjunior@inf.ufes.br; jpalmeida@ieee.org; gguizzardi@acm.org
}

\begin{abstract}
This paper focuses on the issue of ontological interpretation for the ARIS organization modeling language with the following contributions: (i) providing real-world semantics to the primitives of the language by using the UFO foundational ontology as a semantic domain; (ii) the identification of inappropriate elements of the language, using a systematic ontology-based analysis approach; and (iii) recommendations for improvements of the language to resolve the issues identified.
\end{abstract}

Keywords: semantics for enterprise models; organizational structure; ontological interpretation; ARIS; UFO (Unified Foundation Ontology)

\section{INTRODUCTION}

The need to understand and manage the evolution of complex organizations and its information systems has given rise to a number of Enterprise Architecture frameworks in the last decades, including Zachman's framework [27], TOGAF [7], DoDAF [1], MODAF, RM-ODP (with its Enterprise Viewpoint) [6], the ArchiMate framework [5], and the ARIS framework [23].

The majority of these frameworks consider an organization as a system whose elements include: (i) organizational activities structured in business processes and services; (ii) information systems supporting organizational activities; (iii) underlying information technology (IT) infrastructures, and, last but not least, (iv) organizational structures (revealing organizational actors, roles and organizational units).

The relevance of this last domain is clear from a management perspective in that it defines authority and responsibility relations between the various elements of an enterprise and enables one to consider the relations between multiple enterprises. Further, from an IT perspective, organizational actors can be considered system owners, system maintainers, system users or simply system stakeholders in general, affecting the usage and evolution of the enterprise's information systems.

The importance of capturing organizational structures as part of enterprise architecture descriptions has long been recognized in enterprise architecture frameworks. For example, almost two decades ago, organizational structure elements have been included in the people (or "who") column of Zachman's framework [27] and in the organization view of the ARIS Method [23].
Although present in most enterprise architecture frameworks, a semantic foundation for organizational modeling elements is still lacking [1]. This is a significant challenge from the perspective of modelers who must select and manipulate modeling elements to describe an Enterprise Architecture and from the perspective of stakeholders who will be exposed to models for validation and decision making. In other words, a clear semantic account of the concepts underlying Enterprise Modeling languages is required for Enterprise Models to be used as a basis for the management, design and evolution of an Enterprise Architecture.

In this paper we are particularly interested in the modeling of this architectural domain in the widelyemployed ARIS Method (ARchitecture for integrated Information Systems). The ARIS framework is structured in terms of five different views (organization, data, control, function and output) and three abstraction layers (Requirements Definition, Design Specification and Implementation Description) [8, 23]. The organizational view in the requirements definition layer includes modeling concepts for the enterprise's structure (Organizational Unit, Position, Person, etc.) and its own diagrammatic language to produce Organization Charts (which we refer to here as the organizational language).

We focus on the issue of ontological interpretation for the organization modeling elements with the following contributions: (i) providing real-world semantics to the modeling primitives of the organization language by using the well-founded UFO upper-level ontology as a semantic domain; (ii) the identification of inappropriate elements of the organizational language, using ontology-based analysis $([10,11,14,24])$; and (iii) recommendations for improvements of the organizational language to resolve the issues identified (such as ontological mis-interpretations of the language elements and certain usage problems derived from semantic overload and construct redundancy [16]). The interpretation discussed here is complementary to our previous work on a semantic foundation for process modeling in the ARIS method, in which we have addressed the process-related concepts of Event-driven Process Chains (EPCs) [22].

To perform ontological interpretation and analysis, we use concepts of a philosophically and cognitively wellfounded reference ontology called Unified Foundational Ontology (UFO) discussed in depth in $[16,17,18]$ and a 
framework for language evaluation [16]. UFO has been chosen because it unifies several foundational ontologies and has been successfully employed to evaluate, re-design and integrate the models of conceptual modeling languages as well as to provide real-world semantics for their modeling constructs. For example, in [16], a complete evaluation and re-design of the UML 2.0 metamodel using UFO is presented, in [21] ARIS EPCs have been analyzed with UFO and in [1], several enterprise modeling approaches are analyzed with UFO, with a focus on concepts to model rolerelated concepts. An additional example of application of UFO in the analysis and re-design of other modeling languages can be found in [19].

By providing a fuller analysis of the current ARIS metamodel, our work is complementary to the ontological analysis provided by Green, Rosemann and colleagues [4, 10] (see section $\mathrm{V}$ for a detailed discussion on the relation between our approach and the one presented in $[4,10])$.

This paper is organized as follows: section II presents the metamodel for the organizational language, section III introduces the ontological concepts used in our analysis and section IV provides an interpretation for each metamodel element. Section V discusses related work and, finally, section VI presents our conclusions and discusses future work.

\section{THE ARIS ORgAnizATIONAL MetAMODEL}

We adopt here the organizational metamodel which has been excavated in our earlier work [21]. This metamodel defines the abstract syntax of a subset of the language as currently supported by the ARIS Toolset (and thus represents the syntactic elements of the language as currently employed). This metamodel is more up-to-date when compared to the organizational metamodel defined originally by Scheer [23]. The latter includes some elements that are not implemented in the tools (e.g., Object Organization and Profile Organization) and leaves out some of the elements currently supported by the tools (e.g., various metaassociations).

Figure 1 shows a fragment of the excavated metamodel. The main metaclasses for organization modeling language are: Organizational Unit, Organizational Unit Type, Position, Person, Person Type, Group and Location.

As a source of documentation regarding the definitions of the various language constructs, we use the main literature on ARIS ([8], [23]) and the ARIS online documentation, which is the source of our quotes in the remainder of this sub-section.

The Organizational Unit metaclass represents "an entity that is responsible for achieving organizational goals (organization unit)." Examples of organizational units are the "Federal University of Espírito Santo", the "Accounting Department of the Federal University of Espírito Santo", the "Brazilian Federal Senate" and the "Brazilian Chamber of Deputies" (which together make up the "Brazilian National Congress"). An example of Organizational Chart (from [23]) is depicted in Figure 2, revealing the following organizational units: "Sample Co. Inc", its "Sales" Department, its "Billing" and its "Shipping" Department.

The Organizational Unit Type metaclass represents " $a$ type of organization unit, i.e., an element that represents the common features (duties, responsibilities, etc.) of a set of organization units". Examples of Organizational Unit Types are "University", "Federal University", "Federal Senate", "Chamber of Deputies" and "Accounting Department".

The Position metaclass represents "the smallest organizational unit possible. The responsibilities and duties of a position (Position) are defined in the Position

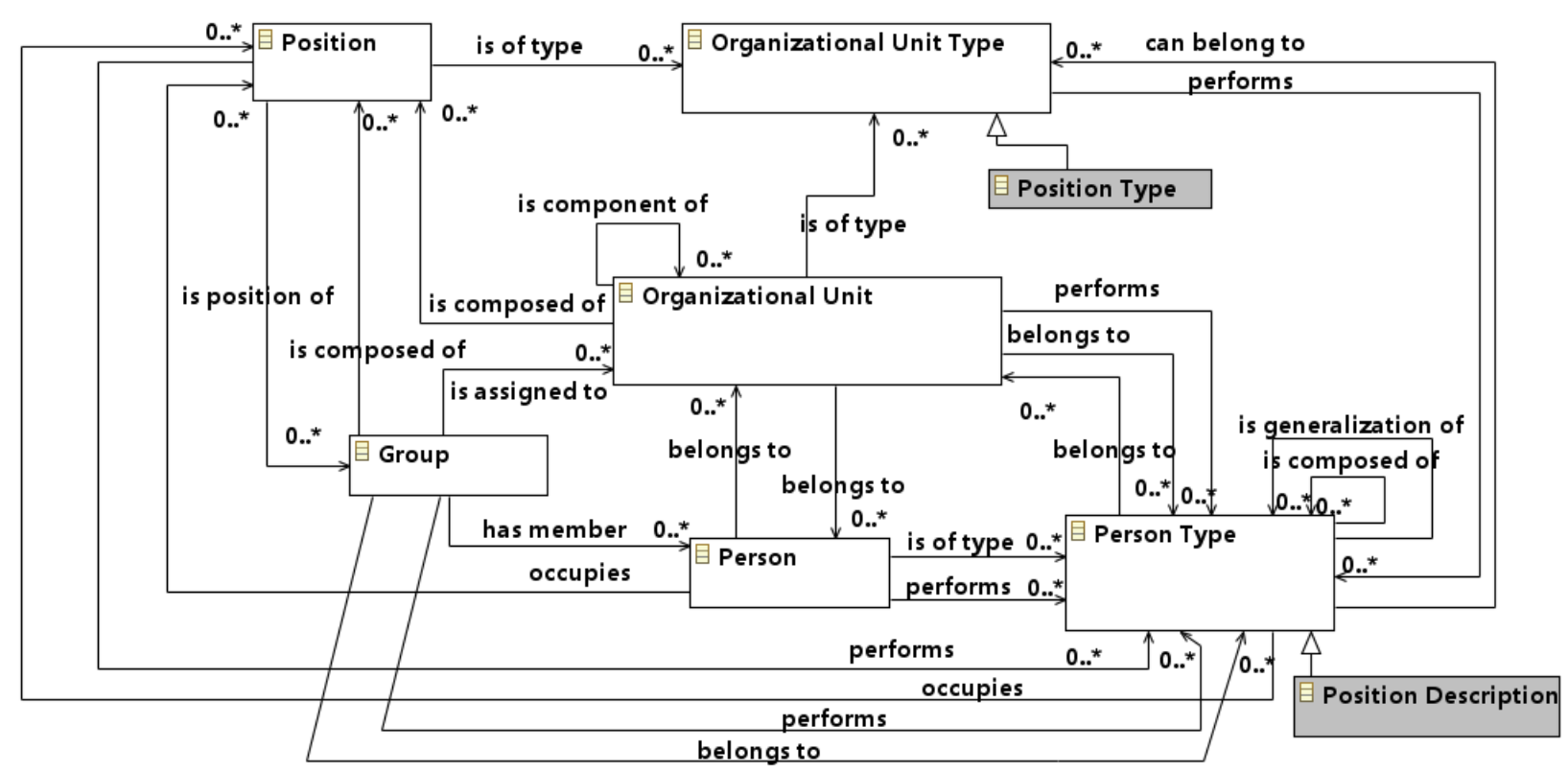

Figure 1. Fragment of Organizational metamodel of ARIS Method 


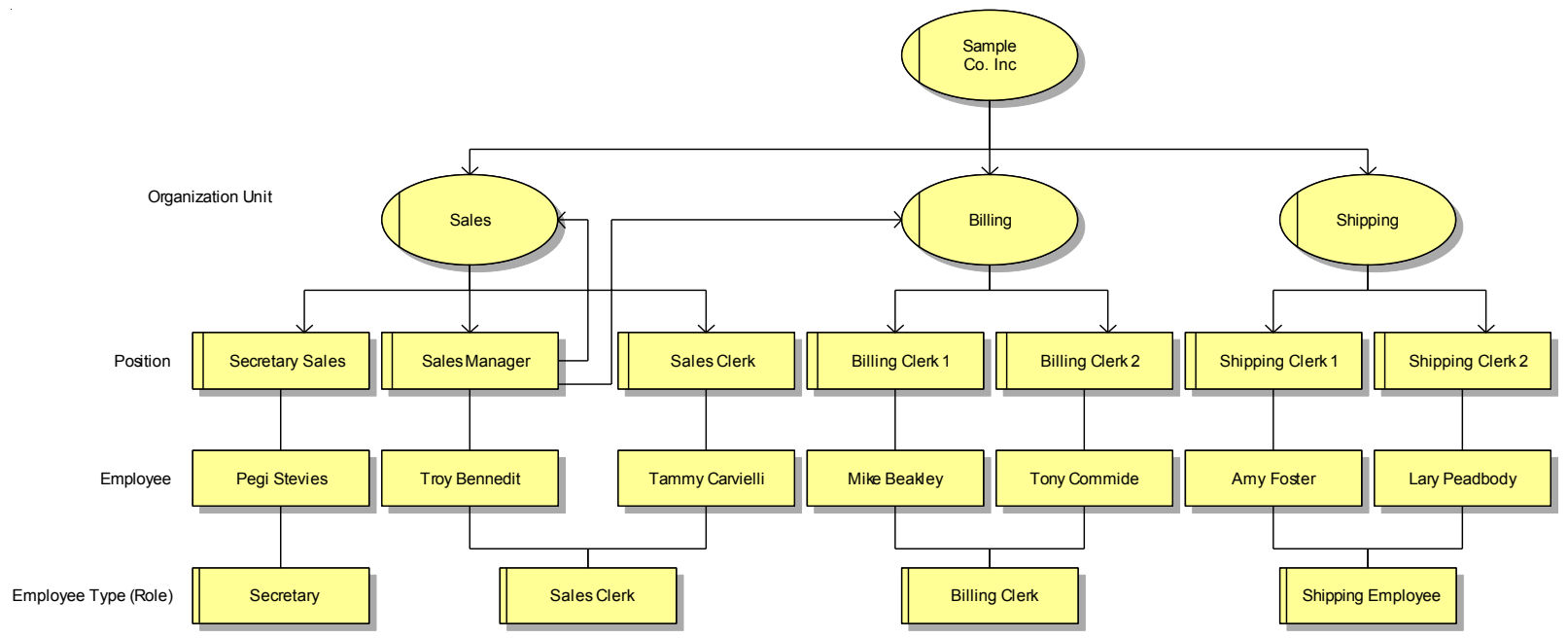

Figure 2. Example of Organizational modeling in Organizational Chart ([23],p.187)

Description". (This is represented here in gray to denote that it is a specialization of Position Type that is applied through the default filter in the toolset.) Examples of Positions include Assistant Professor, Associate Professor, Full Professor, Senator and Accountant. Examples of Positions in an Organizational Chart are shown in Figure 2: "Secretary Sales", "Sales Manager", "Sales Clerk", "Billing Clerk1", "Billing Clerk2", "Shipping Clerk1" and "Shipping Clerk2".

The Position Type metaclass represents a "type of position, i.e. an element that represents the common features (duties, responsibilities, etc.) of a set of positions". Examples include "Professor" and "Member of Congress".

The Person metaclass "is used to represent a person who is assigned to an organization". Examples of Person are "Pegi Stevies", "Troy Bennedit", "Tammy Cavielli", etc. in Figure 2. (Please note that Figure 2 uses outdated terminology for Person and Person Type, calling these elements Employee and Employee Type instead.)

According to on-line documentation of ARIS Toolset the Person Type metaclass represents "a generalization of person, i.e., an element that represents the common features (duties, responsibilities, feature, etc.) of a set of people".

The Group metaclass represents "a group of employees (Person) or a group of organizational units (Organizational Unit) that work together to achieve a goal, e.g., a group of senators and deputies in a parliamentary inquiry committee.

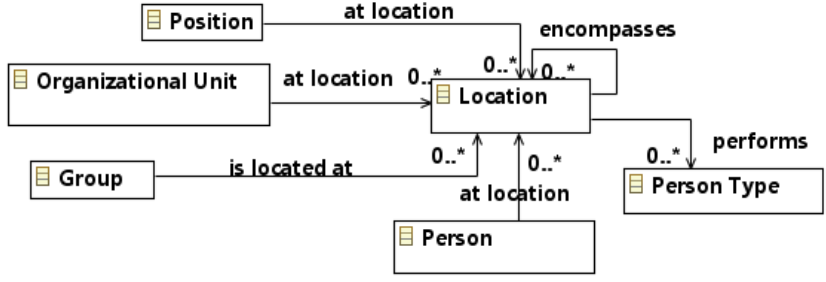

Figure 3. Fragment of the organizational metamodel concerning Location

The Location metaclass (shows in Figure 3) represents " $a$ geographical location of an organization unit, person, position, group, person type". A Location element can represent a region, a city or a building, e.g., "Vitória",
"Brazil", "Brasília" and the "Building of the Brazilian National Congress."

Unfortunately, the on-line documentation of ARIS Toolset and the main literature on ARIS is not explicit about the semantics of the meta-associations present in the organizational metamodel. Thus, we discuss possible interpretations for of each meta-association later in light of the ontological foundations presented in the sequel.

\section{ONTOLOGICAL FOUNDATIONS}

We present briefly a fragment of the Unified Foundational Ontology (UFO) in line with the purposes of this article. For a full discussion regarding this foundational ontology, one should refer to [16].

We start with the fundamental distinction between Universals and Individuals (or Particulars) (see Figure 4). The notion of universal underlies the most basic and widespread constructs in conceptual modeling. Universals are predicative terms that can possibly be applied to a multitude of individuals, capturing the general aspects of such individuals. Individuals are entities that exist instantiating a number of universals and possessing a unique identity.

Further, UFO makes a distinction between the concepts of Endurants and Events (also known as Perdurants). Endurants are individuals said to be wholly present whenever they are present, i.e., they are in time, in the sense that if we say that in circumstance $\mathrm{c} 1$ an endurant e has a property $\mathrm{P} 1$ and in circumstance $\mathrm{c} 2$ the property $\mathrm{P} 2$ (possibly incompatible with $\mathrm{P} 1$ ), it is the very same endurant e that we refer to in each of these situations. Examples of endurants are a house, a person, the moon, the Brazilian Senate and an amount of sand. For instance, we can say that an individual John weights $80 \mathrm{~kg}$ at $\mathrm{c} 1$ but $68 \mathrm{~kg}$ at $\mathrm{c} 2$. Nonetheless, we are in these two cases referring to the same individual John. Events (Perdurants), in contrast, are individuals composed by temporal parts, they happen in time in the sense that they extend in time accumulating temporal parts. An example of an Event is a business process. Whenever an Event occurs, it 


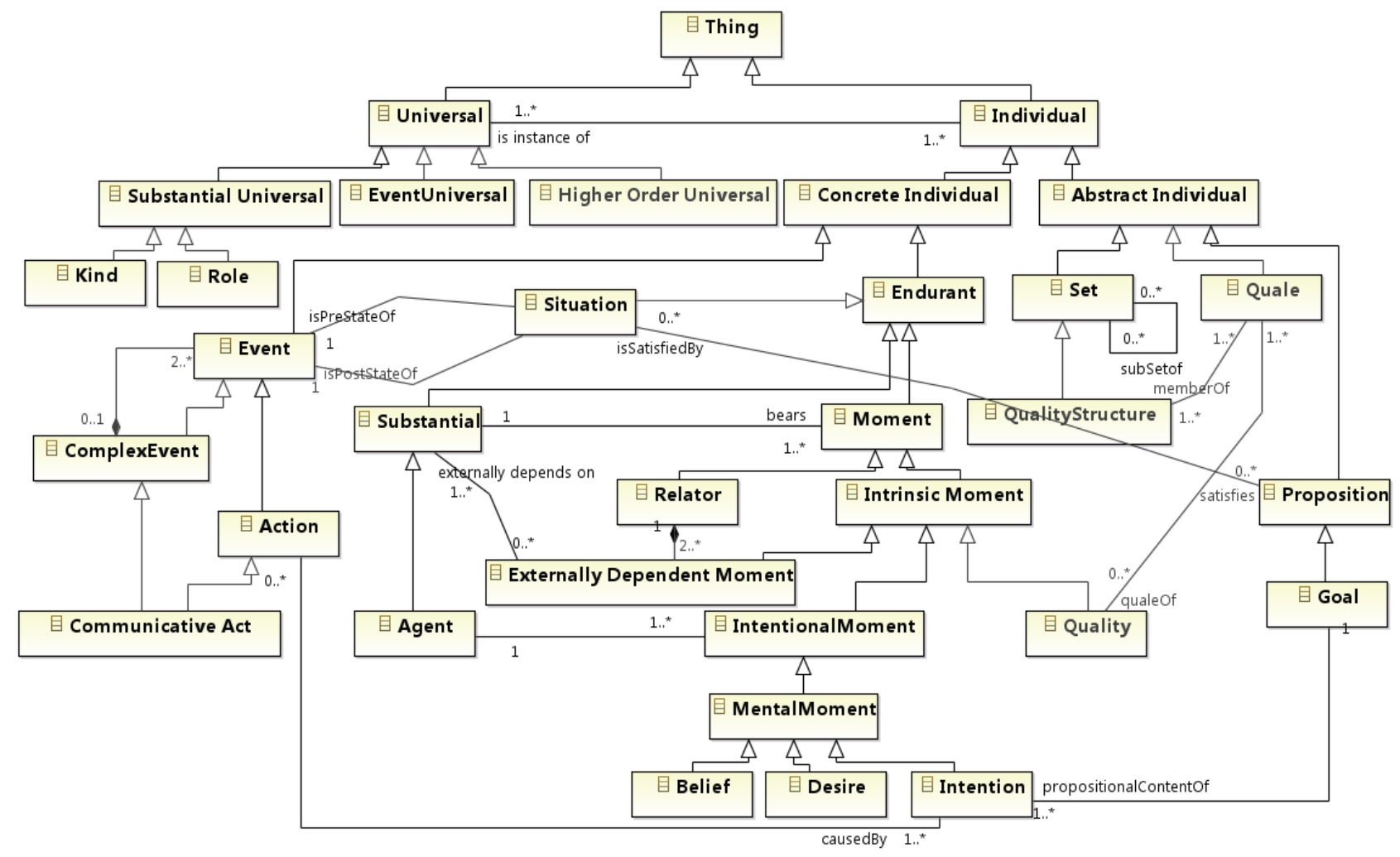

Figure 4 - Fragment of UFO (adapted from [16] and [18])

is not the case that all of its temporal parts also occur. For instance, if we consider a business process "Buy a product" at different time instants when it occurs, at each of these time instants only some of its temporal parts are occurring.

A Substantial is an Endurant that does not depend existentially on other Substantials ${ }^{1}$, roughly corresponding to what is referred by the common sense term "Object". In contrast with Substantials, Moments (also known as particularized properties, objectified properties and Tropes). are existentially dependent entities, i.e., for a Moment $\mathrm{x}$ to exist, another individual must exist, named its bearer. Examples of Substantials include a person, a house, a planet, and the Rolling Stones; examples of Moments include the electric charge in a conductor, a marriage, a covalent bond as well as mental states such as individual Beliefs, Desires and Intentions (or internal commitments). The last three examples fall in the subcategory of Mental Moments.

Existential dependence can also be used to differentiate intrinsic and relational moments: intrinsic moments are dependent of one single individual (e.g., color, a headache, a temperature); relational moments depend on a plurality of individuals (e.g., an employment, a medical treatment, a marriage).

An attempt to model the relation between intrinsic moments and their representation in human cognitive structures is presented in the theory of conceptual spaces introduced in [9]. The theory is based on the notion of

1 Technically, a substantial does not existentially depend on other substantials which are disjoint from it [16].
Quality Structure. The idea is that for several perceivable or conceivable moment universals there is an associated quality structure in human cognition. For example, height and mass are associated with one-dimensional structures with a zero point isomorphic to the half-line of nonnegative numbers. Other properties such as color and taste are represented by multidimensional structures. In [9], the perception or conception of an intrinsic moment can be represented as a point in a quality structure. Following [20], this point is named here a Quale. Quality Structures and qualia are together with Sets, number and Propositions examples of Abstract Individuals. An intrinsic moment universal that is associated with a quality structure is called a Quality Universal, (omitted from Figure 4 due to space constraints) and its instance (an intrinsic moment) is called a Quality.

A kind of externally-independent individual of particular importance to the definition of the concept of role is a "QuaIndividual". An example discussed in [13] clarifies this concept. Suppose that John is married to Mary. John has a number of properties by virtue of being married to Mary. For example, imagine all the legal responsibilities that John has in the context of this relation. These newly acquired properties are moments of John that inheres in him (and are hence existentially dependent on John). However, these moments also depends on the existence of Mary. This type of moment is called externally dependent moment. An externally dependent moment is an intrinsic moment (or quality) that inheres in a single individual but that is existentially dependent on (possibly a multitude of) other individuals external to its bearer (i.e., which is not the 


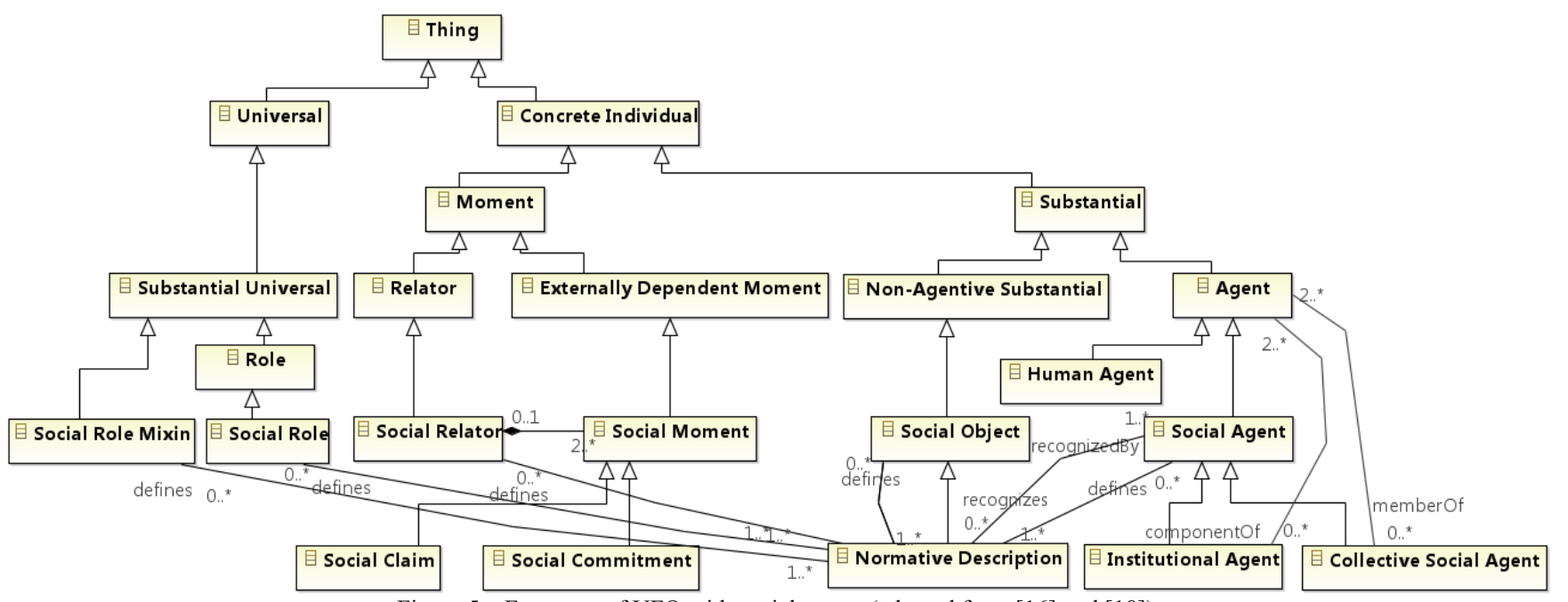

Figure 5. Fragment of UFO with social aspect (adapted from [16] and [18])

bearer's parts or intrinsic moments). In the example, this other individual is Mary.

In the case of an externally dependent moment $x$ there is always an event which is the foundation of $x$. Again, in the given example, we can think of a certain action al (the signing of a social contract) in which both John and Mary participate and which founds the existence of the externally dependent moments inhering in John. Now, we can define an individual that bears all externally dependent moments of John that share the same external dependencies and the same foundation. This individual is called a qua individual [13]. Qua individuals are, thus, a special type of complex externally dependent qualities. In this case, the complex quality inhering in John that bears all responsibilities that John acquires by virtue of the signing of a social contract can be named John-qua-husband.

To continue with the same example, we can think of another qua individual Mary-qua-wife which is a complex moment bearing all responsibilities that Mary acquires by virtue of the same foundation and that albeit inhering in Mary are also existentially dependent on John. The qua individuals John-qua-husband and Mary-qua-wife are existentially dependent on each other. Now, we can define an aggregate composed of these two qua individuals that share the same foundation. This aggregate is called a relator.

A Role universal (Figure 5) applies contingently to an individual that bears (at least one) qua individual of a certain type. (It is also called an anti-rigid universal [16]) We can say that John is not only an instance of a "Person" universal but also an instance of a "Husband" universal, while Mary is both an instance of Person and "Wife" universals. All instances of a "Husband" universal exhibit the behavior required of a husband in a social contract (marriage).

We can say that role universals can be restricted by certain allowed or admissible types, i.e., certain universals to which a role universal can apply. For example, in this case, we can say that the "Student" role can only be played by an instance of the kind "Person". A kind is the substantial universal which supplies a principle of identity for its instances and that is instantiated necessarily by its instances (in other words it is a rigid universal) [16].
The conceptualization in [16] also allows for a notion of Role Mixin Universal which captures commonalities in various role universals. This universal is used in a conceptual modeling design pattern for "roles with multiple disjoint allowed types" (We omit the description of Role Mixins from this paper, please see [16] for a comprehensive discussion and characterization of a role mixin as an antirigid non-sortal universal.). Intuitively, a role mixin universal allows us to add flexibility to a role universal, without tying its definition to a specific kind. For example, it is possible to define a Customer independently of whether Persons or Organizations are allowed to play that role.

UFO also adds distinctions concerning the intentionality of events to this basic core, introducing the notion of Action in this foundation. Actions are intentional events, i.e., events which instantiate a Plan (Action Universal) with the specific purpose of satisfying (the propositional content of) some Commitment of an Agent. The propositional content of a commitment is termed a Goal. Only Agents (entities capable of bearing intentional moments) can perform Actions.

The category of agents further specializes in Physical Agents (e.g., a person) and Social Agents (e.g., an organization, a society). In an analogous manner, objects can also be categorized as Physical Objects (e.g., cars, rocks and threes) or Social Objects (e.g., a currency, a language, the Brazilian constitution). Agents can also be further specialized into Human Agent, Artificial Agent and Institutional Agent, which can be represented, respectively, by human beings, computationally-based agents and an organization or organizational unit (departments, areas and divisions). Institutional Agents are composed by a number of other agents, which can themselves be Human Agents, Artificial Agents or other Institutional Agents.

We should now briefly elaborate on what is meant by stating that "Institutional Agents are composed of other agents". An Institutional Agent exemplifies what is named a Functional Complex in [16], i.e., a mereologically complex entity whose parts play different roles with respect to the whole. By instantiating each of these roles defined in the characterization of that Functional Complex Universal, each part contributes in a different way to the integral behavior of 
the whole. In the case of a social functional complex such as an Institutional Agent, the characterization of the universal instantiated by that agent is made via what is termed in the literature a Normative Description [2].

Each Institutional Agent has a Normative Description associated to it. Moreover, this Institutional Agent defines a context in which a normative description is recognized (see relationship recognizes in Figure 5). We can state then that Normative Descriptions are social objects that create social entities recognized in that context. Examples include Social

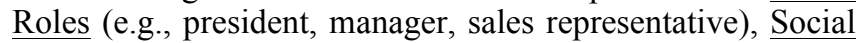
Role Mixins (whose instances are played by entities of different kinds, e.g., customer, which can be played by persons and organizations), Social Agent Universals (e.g., a political party, an education institution), Social Agents (e.g., the Brazilian Labour Party, the University of Twente), Social Object Universals and other Social Objects (e.g., a piece of legislation, a currency) or other Normative Descriptions [1]. A Normative Description that defines social individuals in the context of an institutional agent is termed a Constitutive Normative Description here (and a constitutive norm in [2]).

In addition to Institutional Agents, UFO also acknowledges the existence of Collective Social Agents which are distinguished from Institutional Agents in that all its members play the same role in the collective. An example of a Collective Social Agent is "the group of program committee members which are assigned to review this paper".

Finally, a Higher Order Universal is a universal whose instances are universals. Examples of higher-order universals are "Bird Species" (whose instances could be "Parrot" and "Penguin", both Universals), and "Type of Organization" (whose instances could be "For-Profit Organization" and "Not-For-Profit Organization", also both universals).

\section{ONTOLOGICAL ANALYSIS OF THE ORGANIZATIONAL METAMODEL OF ARIS METHOD}

\section{A. Organization Unit}

The Organizational Unit metaclass in ARIS represents a UFO Institutional Agent. This is because Organizational Units are agentive entities that may be composed of other agentive entities (such as other Organizational Units through the is component of meta-association and, in the end of the decomposition hierarchy Positions as revealed through the is composed of meta-association). These parts (Organizational Units and Positions) play specific roles in this institutional agent, which supports our interpretation.

Organizational Units are "social" agents since they are defined by normative descriptions. In the case of an entire organization (an "enterprise") represented as an Organizational Unit in ARIS this normative description is recognized by the organized society (a Collective Social Agent), which defines what counts as that organization. In the case of a particular sub-division of an organization, this normative description is recognized by the organization and its members.

\section{B. Organization Unit Type}

The Organizational Unit Type element is interpreted as an Institutional Agent Universal, capturing general characteristics of Organizational Units. The is of type metaassociation between Organizational Unit and Organizational Unit Type is interpreted as instantiation. Instantiation is a Formal Relation which occurs between a Universal and a Particular. The sentence ' $p$ is an instance of $U$ ' implies that ' $p$ exemplifies all the properties which are common to all instances of $U$, [16].

Again, Organizational Units Types are "social" universals since they are defined by normative descriptions and are considered to exist for the agents that recognize these normative descriptions.

\section{Position}

According to $[8,23]$, "a position is the smallest organizational unit". If we follow this definition literally, we may be tempted to suggest that a Position should be interpreted as an Institutional Agent (our interpretation for Organizational Unit.)

However, this interpretation is problematic because a Position would be an Institutional Agent which cannot be further decomposed into smaller parts: a Position can only be occupied by a Person. (This can be observed in the metamodel, through the occupies meta-association between Person and Position.) In other words, a Position would be a whole (a Functional Complex) that is composed of only one part (a single Agent), breaking the weak supplementation principle [16]. In other words, why should one distinguish the institutional agent that corresponds to the Position from the actual agent in that Position? [1] Further, it seems that the intention of the language designers was to capture in a Position some general characteristics which are shared by whoever occupies the Position, which seems to suggest an interpretation of Position as some sort of Universal.

To solve this issue, we propose to interpret the Position element as a Social Role which can only be played by a Person (ultimately a Human Agent). In this case, the occupies meta-association between Person and Position is interpreted as instantiation of the Social Role by the Agent. Under this interpretation of the Position element, the problem of weak supplementation is eliminated, because a Position is no longer interpreted as an ontological entity formed by functional parts. (And any Institutional Agent would then be composed of at least two agents.)

The is composed of meta-association between Organizational Unit and Position can be interpreted as capturing the functional composition of an organization unit and one or more positions. At the instance level, this represents a whole-part relationship between the Institutional Agent and whoever instantiates the Social Role (ultimately a Human Agent). This whole-part relation is called componentOf $[15,16]$.

\section{Position Type}

The Position Type element is a notational element in ARIS. This means that it is introduced a posteriori (through a notational filter in the toolset) and thus must be considered 
as a simple specialization of an existing metaclass (in this case an Organizational Unit Type) with no further metaattributes and meta-associations. This is understandable given the ARIS definition of Position as an Organizational Unit. A consequence of this choice in the metamodel is that there is an is of type meta-association between Position and Organizational Unit Type which we believe is intended to be used only for Organizational Unit Types that are specialized into Position Types. We assume here that this is the intention of the tool implementers, and analyze only the relation between Position and Position Type.

Under the suggested interpretation of Position as a Social Role we may interpret a Position Type as: a Social Role or a Higher Order Universal.

In the first case (i), the is of type meta-association between Position and Position Type would be interpreted as subsumption of the Social Role (represented by the Position element) by the Social Role (represented by the Position Type element). An example of this case occurs if we model the Positions "Sales Department Manager", "Engineering Department Manager", "Accounting Department Manager" related to the Position Type "Manager" through is of type.

Under the second interpretation (Position Type as Higher Order Universal), a Position Type characterizes a multitude of Social Roles (universals). In this case the is of type metaassociation between Position and Position Type would be interpreted as instantiation of the Higher Order Universal An example of this case occurs if we model the Positions "Sales Department Manager", "Engineering Department Manager", "Accounting Department Manager" related to the Position Type "Type of Manager" through is of type.

The particular interpretation here depends on the intention of the modeler; we have found plausible examples in usage to suggest either interpretation, constituting a case of semantic overload. We conclude that a revision of the language would be necessary to distinguish between these alternative interpretations. We suggest that the construct be used to denote a Higher Order Universal, since Social Roles can be modeled with the Position construct.

\section{E. Person}

According to the on-line documentation of the ARIS toolset, the Person element represents "a person who may be assigned to an Organizational Unit and Position". This is captured in the metamodel by the belongs to metaassociation between Person and Organizational Unit and by the occupies meta-association between Person and Position.

There are two alternative interpretations here: in the first interpretation, the instances of the Person metaclass represent a particular Human Agent. Under this interpretation, the belongs to meta-association between Person and Organizational Unit can be interpreted as a partwhole relationship (Human Agent is componentOf Institutional Agent). The occupies meta-association between Person and Position can be interpreted as instantiation (in which case the Human Agent instantiates contingently the Social Role universal).

An alternative interpretation is that all instances of the Person metaclass represent Human Agents which instantiate an implicit "Employee" Social Role universal (an interpretation in line with the former name of the Person metaclass: Employee). All Positions in a model would be specializations of this implicit Social Role universal. This interpretation may be undesirable because it would mean that Person (in the ARIS sense) cannot be used to model (external) human stakeholders, relevant to the enterprise model at hand but not an employee of any organization being considered. Thus, in the presence of ambiguity, we recommend the adoption of the first interpretation (Person as a Human Agent) to maximize the applicability of the language ${ }^{2}$.

\section{F. Group}

According to the on-line documentation of ARIS Toolset, the Group element represents a set of employees who are working together for a specific period of time. This suggests that Group represents a whole in a whole-part relation with individuals. We believe it is possible to interpret the Group element as either a Collective Social Agent or as an Institutional Agent. The difference in interpretation will depend on the use of Group element and the associations a Group establishes as a whole.

There are two meta-associations in the metamodel which seem to capture the whole-part relations in which a Group may be involved: is composed of (Positions) and has member (Persons).

If a Group is related to Positions (Social Role) then we should interpret Group as an Institutional Agent. The is composed of meta-association between Group and Position can be interpreted as capturing the functional composition of a group and one or more positions. At the instance level, this represents a whole-part relationship between the Institutional Agent and whoever instantiates the Social Role (ultimately a Human Agent) (as we have discussed earlier this is a wholepart relation called componentOf [16]) An example of this situation occurs when we model a parliamentary inquiry committee in which some of the congressmen play different roles, for example, if one of them is the chairman of the committee. This interpretation of Group renders this concept identical to the concept of Organizational Unit, representing a case of construct redundancy in the language.

However, if a Group is used exclusively to capture a uniform grouping of Persons with no specific roles (i.e., if only has member is used), then we should interpret the Group metaclass as representing a Collective Social Agent. In this case, the has member association should be interpreted as a whole-part relationship called memberOf $[15,16]$. An example of this situation occurs when we model a parliamentary inquiry committee in which all congressmen play the same role. The distinction in interpretation is important given the implications of the different kinds of whole-part relations as discussed in $[15,16]$. In particular,

2 Please observe that this recommendation requires a particular interpretation of "may be assigned" in the quoted ARIS definition, denoting possibility while not implying an obligation or commitment to be assigned to an Organizational Unit and Position (which would characterize a person as an employee). 
memberOf relations are never transitive while transitivity among componentOf relations can hold in certain contexts.

A question that still has to be considered in this last interpretation of Group as a Collective Social Agent is whether the Group represents a collective with an extensional or non-extensional principle of identity. In the case of an extensional principle of identity a change in the composition of the Group would change the Group itself. The nature of the principle of identity cannot be specified in the ARIS organizational language.

A further case of construct deficiency (lack of expressivity; missing construct) can be identified here: there is no notion of Collective Social Agent (Group or other concept) that can be applied to group Institutional Agents (Organizational Units) in ARIS. This would be desirable to capture collectives such as enterprise consortia.

\section{G. Person Type}

According to the on-line documentation of the ARIS Toolset, the Person Type element "is a typification of a set of people who have the same features: responsibilities, rights, obligations, among others". This definition strongly suggests that Person Type should be interpreted as some specific kind of Universal.

Considering the interpretation of Person as Human Agent and the existence of the is of type meta-association between Person and Person Type it could be possible to interpret the Person Type element as a Human Agent Universal. In this case, is of type should be interpreted as necessary instantiation of the rigid Human Agent Universal represented by the Person Type.

However, semantic overloading in the language is revealed when we extend the analysis of Person Type to include all performs meta-associations in which this metaclass participates, namely: the performs metaassociations between Position and Person Type; Person and Person Type; Organizational Unit and Person Type; Organizational Unit Type and Person Type; Group and Person Type; and finally, Location and Person Type. In other words, all metaclasses of the organizational model may perform an ARIS Person Type. (Which is quite surprising given the label "Person Type", which seems to suggest that only "Persons" are characterized by a Person Type.)

To avoid an interpretation in which the performs metaassociations represent an unusually abstract relation that can hold between entities of largely different natures (e.g, capturing both relations between universals and between universals and individuals), we split these meta-associations into two different sets: the performs meta-associations between (i) instance-level elements (Person, Organizational Unit and Group) and Person Type; and (ii) type-level elements (Position, Organizational Unit Type) and Person Type ${ }^{3}$.

Considering the performs meta-associations between instance-level elements (Person, Organizational Unit and Group) and Person Type (i), the most general interpretation

\footnotetext{
${ }^{3}$ We defer interpretations involving Location, since we have not discussed the interpretation of that element yet.
}

for the performs relation is contingent instantiation of the Social Role Mixin represented by the Person Type. This interpretation of Person Type is required when it is used as a universal that captures general contingent characteristics of elements of different natures, in this case, at least, Human Agents (Persons), Institutional Agents (Organizational Units) and Collective Social Agents (Groups). However, it is possible that a particular enterprise model employs Person Type in particular settings to capture general contingent characteristics of elements of specific natures, in which case it is related to either Human Agents (Persons), Institutional Agents (Organizational Units) or Collective Social Agents (Groups). In that case, Person Type should be interpreted as a Social Role. This second interpretation reveals a case of construct redundancy in the language: what would distinguish a Position from a Person Type that is only applied to characterize the contingent behavior of Human Agents (Persons)?

Assuming these two context-dependent interpretations for Person Type (Social Role Mixin or Social Role), we proceed by considering the performs relation between typelevel elements (Position, Organizational Unit Type) and Person Type (ii). The interpretations in this case are also far from trivial, given the flexibility in usage of the elements of the language.

If the relation applies necessarily to all instances of a Position, then we conclude that it should be interpreted as a specialization between the Social Role represented by the Position and the Social Role (Mixin) represented by the Person Type. For example, this occurs if we model that the Position "Senator" (a Social Role) performs the Person Type "Member of Congress" (a Social Role that subsumes the specialized "Senator" Social Role).

However, if it applies contingently to those occupying a Position, then the relation seems to imply that both the Social Role represented by the Position and the Social Role represented by the Person Type share a sortal supertype (a Kind) and further that there is an intersection in the set of instances of the two Social Roles. An example of this situation occurs when we model that the Position "Senator" (a Social Role) may contingently perform the "Member of Parliamentary Committee" Person Type (a Social Role). These Social Roles are non-disjoint specializations of some Human Agent Universal: while some senators may play the role of "Member of Parliamentary Committee" there are "Members of Parliamentary Committee" which are not "Senators" (e.g., "Deputies") and there are "Senators" which are not "Members of Parliamentary Committee". Please note again a case of construct redundancy, since the Social Role "Member of Parliamentary Committee" could be modeled as a Position or a Person Type with the same semantics. When Person Type is interpreted as a Social Role Mixin, then there is an implicit specialization of this Social Role Mixin which shares a sortal supertype (a Kind) with the Social Role represented by the Position. Again, there is an intersection in the set of instances of the two Social Roles.

When the relation applies contingently to the instances of an Organizational Unit Type (Institutional Agent Universal) then there is an unnamed Social Role specializes the 
$\underline{\text { Institutional Agent Universal and the Social Role (or Social }}$ Role Mixin) represented by the Person Type.

If the relation applies necessarily to the instances of an Organizational Unit Type, this would require a different interpretation of Person Type. This is because Person Type can no longer represent a Social Role Mixin, which is, by definition, anti-rigid. In this case, an alternative would be a (Social) Mixin, which is non-rigid and represents properties that are essential to some of its instances and accidental to others [16]. An example which illustrates this situation occurs if we model that an Organization Unit Type "Purchase Department" performs a Person Type "Shopping Client" necessarily and that, at the same time, an Organizational Unit Type "IT Department" may perform the same Person Type contingently (whenever the "IT Department" bypasses the "Purchase Department" and purchases equipment directly.)

In any case, the language lacks expressiveness to distinguish whether the Person Type applies necessarily or contingently to whatever is said to perform the Person Type.

Finally, in all interpretations we consider the is generalization of meta-association between Person Types captures the well-known specialization relation between universals.

\section{H. Position Description}

Similarly to Position Type, Position Description is a notational element in ARIS. Position Description must be considered as a simple specialization of Person Type with no further meta-attributes and meta-associations. We assume that the intention of the tool implementers is to distinguish the case in which a Person Type is used exclusively to characterize Positions (i.e., when only Positions are related to this Person Type through the performs relation.). In this case, the specialized Person Type (Position Description) would simply represent a Social Role, revealing another case of construct redundancy.

\section{Location}

According to the on-line documentation of the ARIS Toolset the Location element represents the geographic location of persons, organizational units, positions and groups. In line with this documentation, we interpret the Location element as representing a Quale that is a member of a Quality Structure to capture geographical notions.

The various meta-associations called is located at and at location are used to associate an implicit Quality of organizational elements (geographical location). For example, through this meta-association it is possible to model that "UFES" (Organizational Unit) is located in "Vitória" (Location). The Location "Vitória" represents a Quale that is a member of a Quality Structure that is a set with all municipalities in Brazil. (Please note that we refrain from a detailed discussion on the semantics of is located at and at location given the many other possible contextspecific interpretations of these relations: consider for example that it may be used to denote allocation of a "Sales Person" to some geographical area, or allocation to a geographical location associated with a particular organizational unit.)

The metamodel also includes an encompasses metaassociation, which allows us to say that a certain location is contained within another location. For example, we can model that the state of Espírito Santo (Location) encompasses the city of Vitória (Location). The encompass relation between Locations should be interpreted as a formal relation that is part of the definition of the Quality Structure. It relates two Quales of the structure, such that the modeler can define a particular Quality Structure suitable to capture the geographical notions for the enterprise architecture at hand.

To proceed with the analysis, we must also consider the performs meta-association. This association seems to suggest that Location is not only establishing geographical notions but is also used as some sort of Organizational Unit. This would constitute a case of semantic overload in the language with very diverging concepts collapsed into the Location element. For example, we could be talking about "Vitória" as an Institutional Agent (in this case the political notion of municipality, which includes a Position of "Mayor") or as a Quale (encompassing all the geographical coordinates within the boundaries of the municipality). In this particular example, there is an organizational counterpart to the geographical location. However, there are many geographical locations which have no organizational counterpart, such as "Room 101 of the Computer Science Building" or "Annex B of the Brazilian Senate Building". These example locations could not possibly perform an intentional role in a business process.

We conclude that the language would be clearer and would have the same expressiveness if the performs metaassociation would be suppressed. Whenever necessary, an Organizational Unit should be defined and related to the corresponding Location through at location.

\section{$J$. Other meta-associations}

We have restricted our analysis to the meta-associations representing instantiation, whole-part relations and specialization. The metamodel also includes a number of meta-associations to enable a modeler to capture notions such as responsibility, cooperation, conflicts, management hierarchy, etc. (These are called substitutes for, is responsible for, is in conflict with, is organizational manager for, cooperates with, is technical superior to, is disciplinary superior to, can be technical superior, is managed by, and have been omitted from the metamodel fragment in Figures 1 and 2.)

Although certain intuitive notions can be inferred from the names of the meta-associations, a precise interpretation for these elements is elusive. Furthermore, the interpretation of these may be highly enterprise-dependent or domaindependent (e.g., consider the different implications of disciplinary superiority in a military setting or in a civilian enterprise, or yet the various kinds of accountability and responsibility constructions in different countries or even different states in the same country.) Therefore, we opt to state only that these represent social relations defined by 
particular normative descriptions in the context in which they apply.

While we focused here on the organizational chart, the modeling elements of the organizational Model are used in several other ARIS Models, for example, the Position, Organizational Unit and Person Type are used in Business Process models (EPC) and Function Allocation Diagram (FAD). Please refer to [22] for an ontological analysis of EPCs using the same foundations discussed here; that work proposes an ontological account for the carries out metaassociation between Function and the organizational elements discussed here (which explains how organizational elements take part in organizational activities).

Table 1 shows a summary of our analysis revealing the possible ontological interpretations we have identified, a diagnosis of language issues, and a suggested ontological interpretation and language recommendations to avoid the issues identified.

\section{DISCUSSION AND RELATED WORK}

In a previous effort [1], we have analyzed several enterprise modeling approaches with UFO, including an initial ontological evaluation of the ARIS role-related concepts. These concepts are discussed here in more depth, although the initial work allows one to position and contrast ARIS with other enterprise modeling approaches.

The study which is most closely related to our work was conducted by Green and Rosemann and presented in [12]. Green and Rosemann discuss an ontological analysis of ARIS models based on the BWW ontology [26]. Similar to our work, Green and Rosemann also conclude that ARIS provides an extensive number of symbols for modelers to choose from that overlap in terms of their real world meanings.

Differently from our work, Green and Rosemann have relied on the metamodels in Scheer's original proposal [23]. As we have discussed in [21], the language metamodel in the ARIS Toolset is significantly different from the metamodels in Scheer's original proposal. As a consequence, the approaches based on Scheer's metamodels do not consider the abstract syntax of the modeling language as actually implemented and employed in enterprises worldwide. (As a consequence some semantic overload issues identified in the work of Green and Rosemann are no longer present in the language.) In addition, our analysis is more specific than that of Green and Rosemann addressing a larger coverage of the individual elements and relations presented in the metamodel.

Other significant differences between the two approaches arise from the choices in the different foundational ontologies employed and the mapping choices employed in the analysis. As we have observed in [22] UFO, but not the BWW ontology, makes an explicit distinction between unintentional events and (intentional) actions. To understand organizations, social roles, business processes and notions such as services as social phenomena, the notions of goals and commitment are of fundamental importance [5]. This requirement places an approach founded on an ontology in which social reality is treated in an explicit manner in clear advantage.

Table 1 - Suggested ontological interpretation and language recommendations for the organizational constructs

\begin{tabular}{|c|c|c|c|}
\hline$\overline{\text { ARIS }}$ & $\begin{array}{l}\text { Possible ontological interpretation (in } \\
\text { UFO) }\end{array}$ & Diagnosis & $\begin{array}{c}\text { Suggested ontological interpretation (in UFO) and language } \\
\text { recommendation }\end{array}$ \\
\hline $\begin{array}{c}\text { Organization } \\
\text { Unit }\end{array}$ & $\underline{\text { Institutional Agent }}$ & - & Institutional Agent \\
\hline $\begin{array}{l}\text { Organization } \\
\text { Unit Type }\end{array}$ & $\underline{\text { Institutional Agent Universal }}$ & - & Institutional Agent Universal \\
\hline \multirow{2}{*}{ Position } & $\begin{array}{l}\text { Social Role which can only be played by } \\
\text { a Person (ultimately a Human Agent) }\end{array}$ & \multirow{2}{*}{ Semantic Overload } & \multirow{2}{*}{$\begin{array}{c}\text { Social Role which can only be played by a Person (avoiding } \\
\text { semantic overload and observing the weak supplementation } \\
\text { principle) }\end{array}$} \\
\hline & Institutional Agent Universal & & \\
\hline \multirow{2}{*}{ Position Type } & Social Role & \multirow{2}{*}{ Semantic Overload } & \multirow{2}{*}{$\begin{array}{l}\text { Higher Order Universal (avoiding semantic overload and } \\
\text { construct redundancy considering the suggested interpretation } \\
\text { for Position) }\end{array}$} \\
\hline & Higher Order Universal & & \\
\hline \multirow{2}{*}{ Person } & Human Agent & \multirow{2}{*}{ Semantic Overload } & \multirow{2}{*}{$\begin{array}{c}\text { Human Agent (avoiding semantic overload and ensuring broad } \\
\text { applicability of the construct) }\end{array}$} \\
\hline & $\begin{array}{l}\text { Human Agent instance of implicit } \\
\text { "employee" Social Role }\end{array}$ & & \\
\hline \multirow{3}{*}{ Person Type } & $\underline{\text { Social Role Mixin }}$ & \multirow{3}{*}{ Semantic Overload } & \multirow{3}{*}{$\begin{array}{l}\text { Social Mixin (non-rigid mixin) (avoiding semantic overload and } \\
\text { construct redundancy considering the suggested interpretation } \\
\text { for Position, while preserving the flexibility in construct use.) }\end{array}$} \\
\hline & $\underline{\text { Social Role }}$ & & \\
\hline & Social Mixin (non-rigid mixin) & & \\
\hline $\begin{array}{c}\text { Position } \\
\text { Description }\end{array}$ & $\underline{\text { Social Role }}$ & Construct Redundancy & $\begin{array}{c}\text { Elimination of the construct to avoid redundancy considering the } \\
\text { suggested interpretation of Position }\end{array}$ \\
\hline \multirow{2}{*}{ Group } & Collective Social Agent & \multirow{2}{*}{ Semantic Overload } & \multirow{2}{*}{$\begin{array}{l}\text { Collective Social Agent (avoiding semantic overload and } \\
\text { construct redundancy considering the suggested interpretation } \\
\text { for Organization Unit) }\end{array}$} \\
\hline & Institutional Agent & & \\
\hline \multirow{2}{*}{ Location } & Quale & \multirow{2}{*}{ Semantic Overload } & \multirow{2}{*}{$\begin{array}{l}\text { Quale (avoiding semantic overload and construct redundancy } \\
\text { considering the suggested interpretation for Organization Unit) } \\
\text { Elimination of the performs } \text { meta-association. }\end{array}$} \\
\hline & $\begin{array}{l}\text { Institutional Agent (when related through } \\
\text { the performs meta-association) }\end{array}$ & & \\
\hline
\end{tabular}




\section{CONCLUSIONS AND FUTURE WORK}

The ontological analysis presented in this paper provides a better understanding of the organizational modeling elements in ARIS with the support of a foundational ontology. An immediate benefit of our ontological analysis is related with the development of organizational models with well-defined real-world semantics. We defend that a clear semantic account of the concepts underlying enterprise modeling languages is key for enterprise modeling to mature as a discipline.

The ontological analysis we have performed has allowed us to reveal problems of usage of certain modeling elements in organizational models, in particular several issues of semantic overload and construct redundancy. The analysis we have performed allows us to justify informal comments in the ARIS literature with respect to the elements of the organizational model. For example, Davis observes when discussing the organizational elements that "it is best to severely restrict the objects available, otherwise people interpret them in different ways" [8].

The interpretation discussed here is complementary to our previous work on a semantic foundation for process modeling in the ARIS method, in which we have addressed the process-related concepts of Event-driven Process Chains (EPCs) [22]. Our next steps with respect to the interpretation of the ARIS method will focus on an ontological analysis of the ARIS notations used for used for capturing enterprise objectives (with some results reported in [3]) and for capturing the detailing of activities (the Function Allocation Diagram - FAD). Our long term objective is the definition of a well-founded subset of the ARIS language for enterprise modeling, accommodating the improvements that arise from ontological analysis.

\section{ACKNOWLEDGMENT}

This research is funded by the Brazilian Research Funding Agencies FAPES (grant number 45444080/09) and CNPq (grants number 481906/2009-6 and 309059/2008-9).

\section{REFERENCES}

[1] J. P. A. Almeida, G. Guizzardi, and P. S. Santos Jr., "Applying and Extending a Semantic Foundation for Role-Related Concepts in Enterprise Modelling", Enterprise Information Systems, vol. 3, 2009, pp. 253-277, doi: 10.1080/17517570903046292.

[2] E. Bottazzi and R. Ferrario, "Preliminaries to a DOLCE Ontology of Organizations", International Journal of Business Process Integration and Management, 2009,vol. 4, no. 4, pp. 225-238.

[3] E. Cardoso, P.S. Santos Jr., J.P.A. Almeida, G. Guizzardi, R.S.S. Guizzardi, "Semantic Integration of Goal and Business Process Modelling", IFIP International Conference on Research and Practical Issues of Enterprise Information Systems (CONFENIS), 2010.

[4] I. G. Davis, M. Rosemann, and P. F. Green, "Exploring proposed ontological issues of ARIS with different categories of modelers", Proc. Australasian Conf. Information Systems, Australia, 2004.

[5] R. Ferrario and N. Guarino, "Towards an Ontological Foundation for Services Science", Lecture Notes in Computer Science, vol. 5468, Springer, 2009.

[6] RM-ODP-ISO-ISO/ITU-T, Open Distributed Processing - Reference Model, 1995.

[7] The Open Group, "The Open Group Architectural Framework (TOGAF 8.1.1 'The Book')", Van Haren Publishing, 2008.
[8] R. Davis, Business Process Modeling with ARIS - A Pratical Guide, Springer, 2001.

[9] P. Gärdenfors, Conceptual Spaces: the Geometry of Thought, USA, MIT Press, 2000.

[10] P. Green and M. Rosemann, "Ontological Analysis of Business Systems Analysis Techniques: Experiences and Proposals for and Enhanced Methodology", In Business Systems Analysis with Ontologies, 2005, pp. 1-27.

[11] P. Green, M. Indulska and M. Rosemann, "A Reference Methodology for Conducting Ontological Analyses", Proceedings of the 23rd International Conference on Conceptual Modelling, 2004 , Shanghai, China, November, ER 2004, pp. 8-12.

[12] P. Green, M. Rosemann, Integrated process modeling: An ontological evaluation, Information Systems, vol. 25 (2), 2000, pp. 73-87.

[13] G. Guizzardi, "Agent Roles, Qua Individuals and The Counting Problem", Software Engineering of Multi-Agent Systems, vol. IV, P. Giorgini, A.Garcia, C. Lucena, R. Choren (eds.), Springer, 2006.

[14] G. Guizzardi, L. Ferreira Pires and M. Van Sinderen, "An OntologyBased Approach for Evaluating the Domain Appropriateness and Comprehensibility Appropriateness of Modeling Languages", ACM/IEEE 8th International Conference on Model Driven Engineering Languages and Systems, Lecture Notes in Computer Science, vol. 3713, Springer, 2005.

[15] G. Guizzardi, "The Problem of Transitivity of Part-Whole Relations in Conceptual Modeling Revisited", Proc. 21st International Conference on Advanced Information Systems Engineering (CAISE'09), Amsterdam, The Netherlands, 2009.

[16] G. Guizzardi, "Ontological Foundations for Structural Conceptual Models", Ph.D. Thesis, University of Twente, The Netherlands, 2005.

[17] G. Guizzardi and G. Wagner, "On A Unified Foundational Ontology and some Applications of it in Business Modeling", Open INTEROP Workshop on Enterprise Modelling and Ontologies for Interoperability (at CAiSE), Latvia, 2004.

[18] G. Guizzardi, R. A. Falbo, and R. S. S. Guizzardi, "Grounding Software Domain Ontologies in the Unified Foundational Ontology (UFO): The case of the ODE Software Process Ontology", XI Iberoamerican Conference on Software Engineering (CIbSE'08), Brazil, 2008.

[19] R. S. S. Guizzardi and G. Guizzardi, "Ontology-Based Transformation Framework from Tropos to AORML", In: P. Giorgini; N. Maiden; J. Mylopoulos; E. Yu. (Org.), Social Modeling for Requirements Engineering, Cooperative Information Systems Series. Boston: MIT Press, in press.

[20] C. Masolo, S. Borgo, A. Gangemi, N. Guarino and A. Oltramari, "Ontology Library", WonderWeb Deliverable D18, 2003.

[21] P. S. Santos Jr., J. P. A. Almeida, and T. L. Pianissolla, "Uncovering the Organizational Modelling and Business Process Modelling Languages in the ARIS Method", International Journal of Business Process Integration and Management (IJBPIM), in press.

[22] P. S. Santos Jr., J. P. A. Almeida and G. Guizzardi, "An OntologyBased Semantic Foundation for ARIS EPCs", Proc. 25th ACM Symposium on Applied Computing (Enterprise Engineering Track), 2010, doi:10.1145/1774088.1774114.

[23] A.W. Scheer, ARIS: Business Process Modeling, 3rd ed., Springer, 2000.

[24] G. Shanks, E. Tansley and R. Weber, "Using Ontology to validate conceptual models", Communications of the ACM, vol. 46, no. 10, 2003, pp. 85-89.

[25] US Department of Defense, "DoD Architecture Framework Version 1.5”, V. II: Product Descriptions, 2007.

[26] Y. Wand, and R. Weber, "An Ontological Model of an Information System", IEEE Transactions on Software Engineering, vol. 16, no. 11, 1990, pp. 1282-1292.

[27] J. Zachman, "Framework for Information Systems Architecture", IBM Systems Journal, Vol. 26, 1987. pp. 276-292. 